\title{
Cesare Beccaria und die Folter \\ - Kritische Anmerkungen aus heutiger Sicht -*
}

Von RiLG Professor Dr. Kai Ambos, Göttingen

\section{Zusammenfassung}

Cesare Beccarias Forderung nach Abschaffung der Folter kann nicht isoliert, sondern nur im Zusammenhang mit seinem legendären Werk „Von den Verbrechen und den Strafen" 1 und der darin zum Ausdruck kommenden kontraktualistisch-utilitaristischen Strafrechtskonzeption gewürdigt werden. Bevor wir uns deshalb seiner eigentlichen Kritik der Folter zuwenden (III.) und ihre aktuelle Bedeutung beurteilen wollen (IV.), ist zunächst die Beccaria-Rezeption im Allgemeinen (I.) und die Entstehungsgeschichte des Werks im Besonderen (II.) zu untersuchen. Die kleine Untersuchung erbringt im Wesentlichen drei Ergebnisse, die ihr zugleich als Thesen vorangestellt werden können:

1. Beccarias Werk hat eine bis heute anhaltende Bedeutung als kriminalpolitisches Manifest zur utilitaristisch begründeten Ersetzung des grausamen, religiösen mittelalterlichen Strafrechts durch ein säkulares und (damit) im Ergebnis humaneres Strafrecht.

2. Beccarias reiht sich insoweit in die Reihe zahlreicher anderer aufklärerischer Denker ein, vermag aber seine - durchaus nicht neuartigen - Thesen so populär zuzuspitzen, dass sie sich rasanter Verbreitung in zahlreichen Sprachen erfreuen. Dies erklärt Beccarias bis heute anbaltende Popularität. Seine fehlende Anerkennung der geistigen Vorläufer seiner Thesen und insbesondere der erheb-

* Erweiterte Fassung eines Vortrags auf dem Alexander von Humboldt Kolleg „Beccaria 250 años después", Santiago de Chile 28.-30. Juli 2010.

1 Ich folge hier der modernen deutschen Übersetzung, die jüngst von Th. Vormbaum besorgt wurde (C. Beccaria, Von den Verbrechen und den Strafen [1764], 2005). Im Übrigen stütze ich mich auf die italienische Urfassung (Dei delitti e delle pene, abrufbar unter www.liberliber.it, 2. elektron. Aufl. 2003) und die darauf beruhende erste spanische Übersetzung von Juan Antonio de las Casas (Tratado de los Delitos y de las Penas, Madrid 1774, abrufbar unter http:// books.google.com. Morellet hat im Jahre 1776 eine völlig neu geordnete französische Übersetzung vorgelegt; vgl. K. Esselborn, Beccarias Leben und Werke, in: C. Beccaria, Über Verbrechen und Strafe, 1905 (Ausgabe und Übersetzung von Esselborn), S. $26 \mathrm{ff}$., der sich nach anfänglicher Kritik (nach Grimm, zit. nach Esselborn, a.a.O., S. 27 mit Fn. ***, war Beccaria zunächst über die Änderungen Morellets so entrüstet, dass er sich nach einem anderen französischen Übersetzer umsah) auch Beccaria in seiner 5. Auflage anschloss (Esselborn, a.a.O., S. 199 ff.) und die sich später nach und nach weltweit durchsetzte, u. a. auch in der in Lateinamerika weit verbreiteten Ausgabe von Temis (Beccaria, De los delitos y de las penas, 3. Aufl. 2003, 5. Nachdruck 2010) und in der hier zugrunde gelegten Übersetzung von Vormbaum (a.a.O.). Die Änderungen Morellets waren zwar nur formaler Natur - die Nummer des Folterkapitels wechselte etwa von XII zu XVI und einige Sätze wurden umgestellt -, doch hat der Text damit an innerer Logik gewonnen, weshalb auch im Folgenden die neuere Fassung zugrunde gelegt wird. 
lichen Mitwirkung der Gebrüder Verri an der Entstehung seines Werks, gerade auch des Kapitels zur Folter, lassen allerdings Zweifel an seiner Originalität und wissenschaftlichen Redlichkeit aufkommen.

3. Die hentige praktische Bedeutung des Werks ist aufgrund der umfassenden normativen (völker- und verfassungsrechtlichen) Absicherung eines humaneren Strafrechts und eines fairen Strafverfahrens als gering zu veranschlagen. Auch per se inhumane und voraufklärerische Strafrechtssysteme bedürfen angesichts der menschenrechtlichen lex lata keiner Beccaria-Lektüre, um von der Notwendigkeit humanisierender Reformen überzeugt zu werden; sie sind zu diesen verpflichtet. Führen sie diese trotzdem nicht durch, wird Beccaria daran auch nichts ändern. Was insbesondere die von Beccaria mit neun Argumenten bekämpfte Folter angeht, so stellt sich die Lage nicht anders dar, wobei freilich seine Konzentration auf die im inquisitorischen Strafverfahren angewendete (repressive) Überführungsfolter den Blick auf die heute diskutierten Probleme im Zusammenhang mit der (präventiven) Rettungsfolter vollkommen verstellt.

\section{Traditionelle und kritische Beccaria-Rezeption}

Für die traditionelle Beccaria-Rezeption wird mit „Dei delitti e delle pene“ das säkulare, humanistische und rechtsstaatliche Strafrecht begründet. Die rechtsstaatlichen Errungenschaften dieses Strafrechts - einerseits die Abschaffung der Todesstrafe und der Folter sowie des geheimen Inquisitionsverfahrens, andererseits die Einführung des Gesetzlichkeitsprinzips ${ }^{2}$ - seien durch dieses Werk maßgeblich bewirkt worden ${ }^{3}$. Diese Ansicht kann vor allem in Lateinamerika als vorherrschend bezeichnet werden. Dort wird das Werk zu den „großen Strömungen“ des Strafrechts gezählt und im gleichen Atemzug mit Autoren wie Carrara, von Liszt u. a. genannt ${ }^{4}$. Vor allem die sog. kritische Kriminologie hat

2 Dazu insbesondere Küper, JuS 1968, 548 f.; krit. aber Naucke, Einführung, in: Vormbaum (Hrsg.) (Anm. 1), S. XIII ff. mit Nachweisen in Fn. 61. Krit. auch schon Naucke (Anm.12) mit Haupttext.

3 Vgl. bezüglich der Todesstrafe etwa E. Weis, Festschrift für Gagnér, 1991, S. 535 ff. („unmittelbare Wirkung ... daß die Todesstrafe und vor allem die Art und Häufigkeit ihrer Anwendung in Zweifel gezogen wurden."). Hinsichtlich der humanistischen Ausrichtung siehe etwa M. Barbero Santos, Actualidad y Derecho (Madrid) No. 11, 18 de marzo de 1990,139ff., 139, 152 (auch in: Centro Nazionale [Anm. 4], S.61 ff.), für den das Werk die Türen zu einem modernen und humanen Strafrecht aufgestoßen hat; auch J.Lekschas, Nachwort, in: K. F. Hommel, Des Herrn Marquis von Beccaria unsterbliches Werk von Verbrechen und Strafen, 1778 (in der von J. Lekschas, 1966 herausgegebenen Ausgabe), S. 223 f.

4 Vgl. z. B. Agudelo B., Estudio preliminar, in: Beccaria, De los delitos (Anm. 1), S. IX f.; ders., in: Centro Nazionale di Prevenzione e Difesa Sociale (Hrsg.), Cesare Beccaria and modern criminal policy, Mailand 1990, S. 401 ff.; Aniyar de Castro, ebd., S. 404 ff.; Beiderman, ebd., S. 414 ff.; Da Costa, ebd., S. 420 f.; Zaffaroni, ebd., S. 422 ff. (der aber einerseits immerhin zugibt, dass Beccaria kein Philosoph gewesen sei, S. 423, und andererseits seinen Einfluss auf das lateinamerikanische Strafrecht nachweist, S. 426 ff.). Tendenziell auch de Faria Costa, 
dem Werk einen nicht nur kriminalpolitischen, sondern auch strafrechtsphilosophischen und -dogmatischen Stellenwert von überragender Bedeutung eingeräumt ${ }^{5}$. So sieht etwa Alessandro Baratta darin „den Ausdruck einer Bewegung, in der die gesamte politische Philosophie der europäischen Aufklärung zusammenfließt“. Es werde damit eine „programmatische Formulierung der Voraussetzungen einer Verbrechens- und Straftheorie sowie des (Straf-)Verfahrens im Rahmen einer liberalen Rechtsstaatskonzeption auf der Grundlage des utilitaristischen Prinzips des maximalen Glücks der größtmöglichen Zahl und des Sozialvertrags und der Gewaltenteilung" vorgelegt ${ }^{6}$.

Gegen diese Ansicht, von Naucke etwas pejorativ als „Beccaria-Schema“ bezeichnet ${ }^{7}$, wendet sich eine Reihe von Autoren, die man eher dem klassischen Strafrecht und der Strafrechtsdogmatik wird zurechnen können und die deshalb vor allem die strafrechtstheoretische Fundierung der Thesen Beccarias kritisch hinterfragen. Diese Autoren erkennen zwar das historische Verdienst Beccarias an, zur richtigen Zeit und am richtigen Ort die Missbräuche des mittelalterlichen, religiös fundierten Strafrechts angeprangert zu haben; sie bezweifeln aber mitunter die Wirkung dieser Kritik und bemängeln häufig ihre fehlende verbrechenstheoretische Fundierung und Systematisierung ${ }^{8}$. So hat schon von Bar in seiner legendären Geschichte des deutschen Strafrechts zwar einerseits das „unbestreitbare[s], unvergessliche[s] Verdienst" Beccarias mit Blick auf die Bekämp-

Boletim da Faculdade de Direito LXXIV, 1998, 89 ff., für den Beccarias präventionsstrafrechtlicher Ansatz „ein wahres kriminalpolitisches Programm“ (101) darstellt.

5 Für eine „Rettung“ (rescate) Beccarias für diese neue Kriminologie sogar Aniyar de Castro (Anm. 4), S. $404 \mathrm{ff}$.

6 A. Baratta, Criminología crítica y crítica del derecho penal, 8. Aufl. 2004, S. 25: „Este tratado es ... la expresión de un movimiento de pensamiento en el que confluye toda la filosofía política del Iluminismo europeo ... La consecuencia de esto para la historia de la ciencia penal ... es la formulación programática de los presupuestos de una teoría jurídica del delito y de la pena, asi como del proceso, en el marco de una concepción liberal del Estado y del derecho basada en el principio utilitarista de la máxima felicidad para el máximo número, y en las ideas del contrato social y de la división de los poderes." (Übersetzung des Verfassers). Zur utilitaristischen Konzeption Beccarias vgl. etwa Neppi Modona, in: Centro Nazionale (Anm.4), S. $77 \mathrm{ff} ., 85 \mathrm{ff}$.

7 W. Naucke, Einführung (Anm. 2); ders., Die Modernisierung des Strafrechts durch Beccaria, in: G. Deimling (Hrsg.), Cesare Beccaria, Die Anfänge moderner Strafrechtspflege in Europa, 1989, S. $37 \mathrm{ff}$.

8 Vgl. schon Esselborn (Anm. 1), S. 17 ff. (S. 19: „Lässt ... auch die theoretische Begründung ... manches vermissen, ... so tut dies dennoch seinem unbestreitbaren und unsterblichen Verdienst keinen Abbruch ..."); Küper, JuS 1968, 547 re.Sp. mit Verweis u.a. auf Frank; G. Kräupl, Die Gesellschaft, der Einzelne und das Verbrechen - Beccarias kriminologisches Verständnis, in: Deimling (Anm. 7), S.155, 161; Th. Vormbaum, Beccaria und die strafrechtliche Aufklärung in der gegenwärtigen strafrechtlichen Diskussion, in: H. C. Jacobs (Hrsg.), Gegen Folter und Todesstrafe, 2007, S. 305, 317 („Respekt für... Bereitschaft zum Nonkonformismus und für... Zivilcourage ..."); zur fehlenden Systematisierung schon K. F. Hommel, Vorrede, in: Hommel (Anm. 3), S. 7; auch J. Llobet R., Garantías y sistema penal. Releyendo hoy a Cesare Beccaria, 1999, S.33. Für eine generelle Kritik an der Aufklärung insoweit Vormbaum, a.a.O., S. 317 („,aufklärerische Kritik“ an der Strafrechtstheorie der Aufklärung); M. Cattaneo, Aufklärung und Strafrecht, 1998, S. 42. 
fung der Missbräuche des damaligen Strafrechts anerkannt ${ }^{9}$, andererseits aber über eine „populär[e]“ Darstellung hinaus „nichts Neues“ in der Schrift entdecken können ${ }^{10}$ und vor allem auch die „Folgerichtigkeit und Genauigkeit“ der theoretischen Begründung bemängelt ${ }^{11}$. Radikaler noch sieht Naucke in Beccaria zwar den Kritiker des alten, abgelebten, religiös begründeten Strafrechts, doch zugleich auch den Begründer bzw. Verstärker eines an Effektivität und gesellschaftlichem Nutzen orientierten neuen (harten) öffentlichen Strafrechts, bei dem lediglich die religiöse Begründung des Strafrechts durch die säkulare ersetzt werde ${ }^{12}$. Beccarias Forderungen - Abschaffung der Todesstrafe und der Folter - und seine Prinzipien - Verhältnismäßigkeit, Gesetzlichkeit ${ }^{13}$, Sozialschädlichkeit - seien zu oberflächlich und schlecht begründet, um die unverrückbaren rechtsstaatlichen Grenzen eines humanen Strafrechts fixieren zu können ${ }^{14}$. Seine allgemeinen Lehren seien zu wenig konkret, sein „Gefühl für Humanität"15 „irrational“ und zu „wenig inhaltsreich“, um gegen die inhumanen Entgleisungen des Strafrechts in seiner konkreten Ausgestaltung und Anwendung ankommen zu können ${ }^{16}$. Dazu bedürfe es eben nicht eines herzensguten, aufgeklärten (säkularen) Juristen, sondern des „in den juristischen Techniken gut ausgebildete[n], aber gegen jede Herrschaftsform mißtrauische[n] Jurist[en] (den es bei Beccaria nicht gibt) "17. Beccarias „unvermeidlicher" Sozialvertrag, dem sich niemand entziehen könne, führe zur Unvermeidbarkeit und

9 L. von Bar, Handbuch des deutschen Strafrechts. Erster Band: Geschichte des deutschen Strafrechts und der Strafrechtstheorien, 1882, S. $234 \mathrm{f}$. In diesem Sinne sprach auch schon Hommel (Anm. 3) im Jahre 1778 von dem „unsterblichen“ Werk Beccarias.

10 Vor allem nicht mit Blick auf die Ablehnung eines göttlich begründeten Strafrechts (von Bar [Anm.9], S.235). Dazu auch Küper, JuS 1968, 550 li.Sp., 551 li.Sp., womit Beccaria das Strafrecht auf eine „neue rationale Basis gestellt" habe.

11 Einerseits hinsichtlich der „oberflächlichen und verkehrten Ansicht“, dass die „Strafgesetze einer wissenschaftlichen Interpretation entbehren könnten“; v. Bar (Anm. 9), S. 233; andererseits hinsichtlich der vertragsrechtlichen und generalpräventiven Begründung der Strafe, weil sie auf der „Fiction der Einwilligung" in die Strafe und der Aufopferung zur Abschreckung anderer beruhe (ebd.; vgl. insoweit schon I. Kant, Metaphysik der Sitten, 1797, Ausgabe Meiner 3. Aufl. 2009, S. 155, der bekanntlich Beccaria und allgemein den Vertretern der Präventionslehren vorwirft, den Mensch „unter die Gegenstände des Sachenrechts“ zu mengen, also als Mittel zu den Zwecken von Staat und Gesellschaft benutzen zu wollen; dazu auch Küper, JuS 1968, 550 re. Sp.) und diese Einwilligung nicht partiell nur bzgl. der Todesstrafe zurückgenommen werden könne (aufgrund einer „partiellen“ Vertragstheorie, Küper, JuS 1968, 552 re. Sp.), weil ja auch andere Freiheitsstrafen äußerst grausam und damit nicht einwilligungsfähig waren (so in der Sache von Bar [Anm. 9], S. 234). Krit. insoweit auch Küper, JuS 1968, $552 \mathrm{f}$., für den das wesentliche Gegenargument der Irreparabilität der Todesstrafe fehlt; auch Naucke, Einführung (Anm. 2), S.XXVf., für den die Begründung Beccarias von „politisch fahrlässiger Oberflächlichkeit“ ist und seine alternative Freiheitsstrafe die „säkularisierte Hölle“.

12 Naucke, in: Deimling (Anm. 7), S. 42 ff., 42, 50; ders., Einführung (Anm. 2), S.XLf.

13 Ein absolut verstandenes Gesetzlichkeitsprinzip führe zu einer grenzenlosen Gesetzesmacht, der sich der Bürger unterwerfen müsse; Naucke, in: Deimling (Anm. 7), S. 49 f.; ders., Einführung (Anm. 2), S.XXIXf.

14 Naucke, Einführung (Anm. 2), S.XXVff.

15 Ebd., S. XIX.

16 Ebd., S.XXIIff.

17 Ebd., S. XXIV. 
Notwendigkeit des Strafens und damit zu einem noch mächtigeren Strafrecht ${ }^{18}$. Beccarias utilitaristische Begründung des Strafrechts habe zur Folge, dass die von ihm propagierten Prinzipien „nicht also absolute Grenzen jeden Strafens, sondern ... als Unterstützungen der Zweckmäßigkeit des Strafens“ diskutiert würden ${ }^{19}$, es geht also um Begründung (Legitimation), nicht Begrenzung (Limitation) des Strafrechts. Überdies sei der utilitaristische Ansatz austauschbar: Er diene nicht nur einem liberalem, vernüntigen Strafrecht, sondern auch allen anderen Strafrechten, vom human-liberalen bis zum inhuman-brutalen ${ }^{20}$ und die jeweilige Macht könne, dank der „nur mit Willkür zu füllenden Generalklausel“ 21 der Sozialschädlichkeit, selbst entscheiden, wie viel und welches Strafrecht sie zu ihrer Erhaltung brauche ${ }^{22}$. So erweise sich der strafrechtliche Rechtsstaat Beccarias als der "gesetzlich verstärkte Sicherheitsstaat mit wechselnden Inhalten “ 23 . Beccarias Strafrecht sei alles, vor allem säkular, aber „[h]uman ist es nicht“24.

Diese scheinbar gegenläufigen Ansichten lassen sich in Übereinstimmung bringen oder doch einander annähern, wenn man das Werk Beccarias als das nimmt was es ist, nämlich als ein kriminalpolitisches Manifest oder Programm ${ }^{25}$, dessen - durch die rasante Verbreitung in mehreren Sprachen dokumentierter ${ }^{26}$ Erfolg gerade in der thesenartigen und populären ${ }^{27}$ Zuspitzung rechtsstaatlicher, im Zuge der Aufklärung nicht länger aufschiebbarer und zur damaligen Zeit ${ }^{28}$ wahrlich revolutionärer Kernforderungen zu sehen ist. Ein darüber hinausgehender theoretischer Anspruch ist mit einem solchen Manifest nicht verbunden, denn die von Beccaria propagierte „utilitaristische[n] Fundierung des Kontraktualismus“" 29 stellt eine ( $\mathrm{zu}$ ) dünne philosophische Grundlage dar, um darauf eine konkrete Verbrechenslehre errichten zu können ${ }^{30}$. Vor allem aber führt seine

18 Naucke, in: Deimling (Anm. 7), S. 45; ders., Einführung (Anm. 2), S. XLI.

Naucke, in: Deimling (Anm. 7), S. 50.

Naucke, in: Deimling (Anm. 7), S. 52; ders., Einführung (Anm. 2), S. XLI.

Ebd., S.XXXI.

Ebd., S. XXXI.

Ebd., S. XLII.

Ebd., S. XLIII.

5 Ähnlich de Faria Costa (Anm. 4), 91 und passim („programa de política criminal“); E. Malarino, Pietro Verris „Betrachtungen über die Folter“ und die Debatte über die Abschaffung der Folter in der österreichischen Lombardei, in: Th. Vormbaum (Hrsg.), Pest, Folter und Schandsäule, 2008, S. 171, 197 („Pamphlet“).

26 Weis, Festschrift für Gagnér, 1991, S. 538 („Echo wie nur wenige Bücher der Geistesgeschichte").

27 V. Bar (Anm. 9), S. 235. Aniyar de Castro (Anm. 4), S. 407 spricht insoweit von einer „literarischen Eleganz der Sprache Beccarias“ („elegancia literaria del lenguaje beccariano“).

28 Instruktiv zum deutschen Kontext eines feudal-absolutistischen Strafrechts Lekschas (Anm. 3), S. $229 \mathrm{ff}$.

29 W. Rother, Zwischen Utilitarismus und Kontraktualismus: Beccarias Kritik an der Todesstrafe im philosophischen Kontext, in: Jacobs (Anm.8), S.186. Instruktiv dazu Küper, JuS 1968, $548 \mathrm{ff}$.

30 Vgl. auch G. Deimling, Gedächtnisschrift für H. Kaufmann, 1986, S. 63 wonach die kriminalpräventiven Forderungen Beccarias nicht auf einer „expliziten Verbrechenstheorie“ beruhten, sondern sich seine Einteilung der Verbrechen und Strafen ausschließlich nach dem zugefügten gesellschaftlichen Schaden richte; dazu auch Küper, JuS 1968, 550 li. Sp. 
„eigentümliche Kombination humanitärer Forderungen mit utilitaristischen Zweckerwägungen " $31 \mathrm{zu}$ offensichtlichen Widersprüchen ${ }^{32}$, die letztlich nur durch die Zurückstellung der durchaus vorhandenen humanitären Erwägungen $^{33}$ hinter Gemeinwohlinteressen aufgelöst werden können. Das wird etwa an seinen Ausführungen zur Todesstrafe ${ }^{34}$ deutlich, die er letztlich nur aus Nützlichkeitserwägungen ablehnt: zum einen befürwortet er sie, wenn „sie andere von der Begehung von Verbrechen" abhalten würde ${ }^{35}$; zum anderen fordert er aus diesen generalpräventiven Effizienzerwägungen heraus noch grausamere Freiheitsstrafen ${ }^{36}$. Vor allem aber steht die "Gewißheit und Unverbrüchlichkeit der Strafen“ für Beccaria nicht zur Disposition, denn dies entspreche zwar der Menschlichkeit, widerspreche aber dem Gemeinwohl, dessen vornehmster Ausdruck das „Recht zum Strafen“ sei ${ }^{37}$; Vergebung und Gnade hingegen nährten nur „die Verlockung der Straflosigkeit“ und schwächten damit das Strafrecht ${ }^{38}$. Eine Strafe sei gerecht, wenn sie notwendig sei ${ }^{39}$. Immerhin kann man dem am

31 Küper, JuS 1968, 551 re. Sp.: Humanität nicht als Selbstzweck sondern als Gebot der Vernunft. Anders ausgedrückt: nur humanitäres Strafrecht ist vernünftig, weil effizient und damit nützlich. Vgl. auch Naucke, in: Deimling (Anm. 7), S. 44, der in „Humanität und Effektivität“ die Gründungen des Beccariaschen Strafrechts sieht, wobei das Humanitätsargument letztlich utilitaristischen Überlegungen untergeordnet werde, weil Humanität nur „als Mittel der Kritik an einer unzweckmäßigen Strafrechtspflege“ diene (S. 47 ff., 49). Humanität und Effektivität müssten aber, wenn man Strafrecht begrenzen wolle, getrennt werden (S. $52 \mathrm{f}$.).

32 Vgl. etwa Beccaria, Von den Verbrechen (Anm. 1), S. 28 f. (Kap. X. Suggestivbefragungen. Aussagen), wo er es einerseits als „naturwidrig“ bezeichnet, dass „ein Beschuldigter sich unmittelbar selbst beschuldigt“, er andererseits aber denjenigen, „der beim Verhör hartnäckig die Antwort auf die ihm gestellten Fragen verweigert" streng bestrafen will; krit. dazu Jerouschek, ZStW 110 (1998), S. 671, für den sich dahinter das „Medusenhaupt des Geständniszwangs ... in generalpräventivem Gewand“ zeige. Oder Beccaria, a.a.O., S. 48 ff. (Kap. XVI. Von der Todesstrafe), wo er einerseits den Kampf gegen die Todesstrafe auch als Kampf für Menschlichkeit bezeichnet (S. 49), andererseits aber sogar grausamere Freiheitsstrafen als die Todesstrafe aus (general-)präventiven Gründen fordert (S. 50: „zu solchen langdauernden und elenden Lebensbedingungen erniedrigt ...“; S. 52: „.. . in eisernem Käfig . . . beim Zusammenzählen aller unglücklichen Augenblicke der Knechtschaft diese vielleicht sogar noch schmerzhafter“ als die Todesstrafe „ist“; ... „und ebendies ist der Vorteil der Strafe der Knechtschaft, die denjenigen, der ihr zuschaut, mehr in Schrecken versetzt als denjenigen, der sie erduldet ...") und wiederum widersprüchlich die Todesstrafe gerade wegen ihre Grausamkeit als „nicht nützlich“ erachtet (S.54). Für Mondolfo, Cesare Beccaria, 1960, S.65 (zit. nach Cattaneo [Anm. 8], S. 47) hat Beccaria den Widerspruch zwischen humanistischem Prinzip und utilitaristischer Begründung der Strafe nicht erkannt.

33 S. vor allem Beccaria, Von den Verbrechen (Anm. 1), S. 77: „Wo die Gesetze erlauben, daß der Mensch unter gewissen Voraussetzungen aufhört, Person zu sein, und zur Sache wird, dort gibt es keine Freiheit.“ (in: Dei delitti [Anm. 1], Kap. XX, S. 26). Für Agudelo B., in: Centro Nazionale (Anm. 4), S. 402 f. zeigt sich darin Beccarias Übereinstimmung mit Kant hinsichtlich seines Bekenntnisses zur Menschenwürde, die eine Vermengung des Menschen mit den Sachen (Anm. 11) verbiete.

34 Vgl. schon Anm. 32. Zur Rezeption im 18. Jh. insoweit B. Krentziger, Argumente für und wider die Todesstrafe(n), in: Deimling (Anm. 7), S. $99 \mathrm{ff}$.

35 Beccaria, Von den Verbrechen (Anm. 1), S. 49.

36 Vgl. schon Anm. 32.

37 Vgl. Beccaria, Von den Verbrechen (Anm.1), Kap. XX. Gewißheit und Unverbrüchlichkeit der Strafen. Gnade, S. 64 („Recht zum Strafen ist ... das aller Bürger oder des Herrschers“).

38 Ebd., S. 65.

39 Ebd., S. 98. 
Gesellschaftsvertrag und an der Sozialschädlichkeit orientierten Strafrechtsverständnis Beccarias einen - für die bürgerliche Aufklärung des 18. Jahrhunderts allerdings typischen ${ }^{40}$ - objektivistischen Verbrechensbegriff ${ }^{41}$ und einen ausbaufähigen Ansatz für eine materielle Bestimmung des Unrechts entnehmen ${ }^{42}$. So lässt sich in der heute herrschenden Rechtsgutslehre durchaus die Konkretisierung der Sozialschädlichkeitslehre sehen ${ }^{43}$, was freilich nichts daran ändert, dass diese Lehre, vor allem in ihrer realsozialistisch-sowjetischen Ausprägung der Sozial- oder Gesellschaftsgefährlichkeit ${ }^{44}$, zum Einsatz des Strafrechts als politisches Disziplinierungsinstrument der „Konterrevolution“ missbraucht werden kann ${ }^{45}$ und deshalb die Skepsis Nauckes ${ }^{46}$ - trotz ihres zugleich anzuerkennenden Limitierungspotentials ${ }^{47}$ - durchaus berechtigt ist. Wie dem auch sei, man wird auch sagen können, dass Beccaria das Strafrecht mit seiner Befreiung von religiösen Elementen und der Orientierung am Gesellschaftsschaden bzw. Gemeinwohl jedenfalls „auf eine neue rationale Basis gestellt“ ${ }^{48}$ und „im Diesseits der sozialen Ordnung angesiedelt" hat ${ }^{49}$, wenn auch humanistischen Erwägungen dabei allenfalls sekundäre Bedeutung zukam ${ }^{50}$, will man diese nicht im

40 Vgl. Lekschas (Anm. 3), S. $250 \mathrm{ff}$.

41 Vgl. Beccaria, Von den Verbrechen (Anm. 1), Kap. XXIV. Maßstab der Verbrechen, S. 72: der wahre Maßstab der Verbrechen sei „Schaden für die Gesellschaft“, nicht „die Absicht dessen, der sie begeht.“ Dazu auch jüngst L. Gracia Martín, GA 2010, 323, 334 (Sozialschädlichkeit aufgrund Verletzung der Vertragsbedingungen).

42 Küper, JuS 1968, 550 li.Sp., 551 re.Sp. m.w.N.

43 Vgl. jüngst etwa $N$. Wrage, Grenzen der staatlichen Strafgewalt: Überlegungen zu einer Renaissance des materiellen Verbrechensbegriffes, 2009, S. 42 ff., der von einer Typisierung durch die Rechtsgutslehre ausgeht. Vgl. auch Y. Meng, Die Strafbarkeit der Vorbereitungshandlung des Delikts nach dem deutschen und chinesischen StGB, 2009, S. 114: „Die Rechtsgutslehre öffnet eine Tür zum Nachweis der Sozialschädlichkeit, wodurch das Rechtsgüterschutzprinzip der Verhinderung der sozialschädlichen Handlungen dient." Zur systemtheoretischen Nutzung der Sozialschädlichkeit als materialer Maßstab bei Amelung und Jakobs jüngst (krit.) Swoboda, ZStW 122 (2010), S.24, 41 ff. m.w.N.

44 Vgl. Lammich, ZStW 109 (1997), S. 417, 422 f.; Rinceanu, ZStW 121 (2009), S. 792, 802 ff.

45 Krit. etwa Lammich, ZStW 109 (1997), S. 423; Rinceanu, ZStW 121 (2009), S. 806 f. Vgl. auch $\$ 13 \mathrm{c}$ chinesStGB (der auf $\$ 7$ sowjetStGB beruht), wonach Verbrechen u. a. ,alle sozialschädlichen Angriffe“ gegen die „volksdemokratische Diktatur, das sozialistische System“ sind (zit. nach Yang/Richter, Begriff und Systematisierung der Straftat in China, in: Sieber/Cornils [Hrsg.], Nationales Strafrecht in rechtsvergleichender Darstellung. Teilband 2, 2008, S. 353).

46 Oben Anm. 21 und Haupttext.

47 Vgl. nochmal $\$ 13 \mathrm{c}$ chinesStGB, aus dem sich die limitierende Funktion der Sozialschädlichkeit bei Handlungen ergibt, die „wegen Geringfügigkeit keine große Sozialschädlichkeit“ darstellen und deshalb nicht als Verbrechen gelten (sog. Bagatellklausel); dazu auch Yang/ Richter (Anm. 45), S. 354; Rinceanu, ZStW 121 (2009), S. 802 ff.; zum geltenden polnStGB (Art. 1 \S 1-3) insoweit Weigend/Wróbel, ZStW 122 (2010), S. 259, 260.

48 Küper, JuS 1968, 551 li.Sp. Zur antireligiösen Stoßrichtung schon Hommel (Anm. 8), S.2 („Man muß Sünde, Verbrechen und verächtliche Handlungen nicht unter einander werfen“), 10 („Gottes Gerichte und menschliche Gerichte sind heterogene Dinge und so schwerlich wie Wasser und Öl mit einander zu vermischen, weil ihre Bestandteile und ihre Quellen verschiedentlich sind."); auch Vormbaum (Anm. 8), S.317.

49 Küper, JuS 1968, 551 li.Sp.

50 Positiver insoweit Küper, JuS 1968, 551 li.Sp. („als menschliche Institution im Dienste menschlicher Zwecke“, hier weggelassener Teil des Zitats bei Anm. 49 mit Haupttext). 
Primat der Verbrechensverhütung ${ }^{51}$ verwirklicht sehen. Im Zweifel geht es Beccaria also nicht um ein per se humanes und gerechtes, sondern ein effizientes und sozial nützliches Strafrecht ${ }^{52}$.

Mit diesen Einschränkungen stellt Beccarias „Büchlein“ (libricino), richtig verstanden als kriminalpolitisches Manifest ${ }^{53}$, einen Meilenstein der Aufklärung ${ }^{54}$ und letztlich der - jedenfalls normativen - Abschaffung der Folter dar ${ }^{55}$. Es dient nach wie vor als Fixpunkt des klassisch-liberalen Strafrechts ${ }^{56}$, unabhängig davon, ob man dieses zur Lösung der heutigen Probleme der globalisierten Risikogesellschaft für geeignet hält oder nicht ${ }^{57}$. Eine kritische Lektüre verdient das Werk damit auch heute noch, vor allem in Ländern mit einem antiaufklärerischen Strafjustizsystem ${ }^{58}$.

\section{Zur Entstehung von „Dei delitti e delle pene“}

Gravierender als die dogmatische oder theoretische Kritik an der Schrift erscheint denn auch ihre Entstehungsgeschichte, ergibt sich doch daraus, dass Beccarias (alleinige) Urheberschaft - wohl weniger aus intellektuellen als vielmehr aus Gründen mangelnden Arbeitseifers - ernsthaft in Zweifel gezogen werden muss. Aus der jüngst von Malarino vorgelegten, äußerst gründlichen Analyse der Korrespondenz der Gebrüder Verri und der einschlägigen italienischen Beccaria-Forschung ergibt sich in kaum zu überbietender Eindeutigkeit, dass insbesondere Pietro Verri maßgeblich zur Entstehung des Werks beigetragen

51 Beccaria, Von den Verbrechen (Anm. 1), Kap. XLI. Wie man Verbrechen verhütet, S. 107 ff., 107: „Es ist besser, Verbrechen zu verhüten, als sie zu bestrafen“.

52 W. Alff, Einführung, in: ders. (Hrsg.), Über Verbrechen und Strafen (Übersetzung nach der Ausgabe von 1766), 1988, S. 39 weist darauf hin, dass bei Beccaria das Wort „Humanität“ gar nicht vorkomme, sondern lediglich „Empfindsamkeit“ (sensibilità) i. S.v. Empfänglichkeit von Lust und Schmerz. Anders sieht Cattaneo (Anm. 8), S. 42 ein humanes Strafrechtsverständnis neben präventiver Straftheorie und, S.47, ein Bekenntnis zur Menschenwürde. In diesem Sinne auch Pisapia, in: Centro Nazionale (Anm.4), S.60; G.O.W. Mueller, ebd., S. 102; Delmas-Marty, ebd., S. 141; Agudelo B., ebd., S. 402 f.

53 Vgl. schon Anm. 25.

54 Zum „bedeutenden Aufschwung“, den die deutsche Aufklärung durch die Übersetzung des Werks nahm siehe Lekschas (Anm. 3), S. 242.

55 Malarino (Anm. 25), 197.

56 Vgl. zuletzt Schünemann, GA 2010, 353, 359.

57 Zum Widerstandsdiskurs der sog. - so gar nicht existierenden - Frankfurter Schule versus modernen Positionen eines Strafrechts der zwei Geschwindigkeiten oder eines sozialen und demokratischen Strafrechts, das gerade auch kollektive Rechtsgüter schützen will, zuletzt J.M. Silva Sanchez, GA 2010, $307 \mathrm{ff}$. und Gracia Martin, GA 2010, $323 \mathrm{ff}$.

58 So erklärt sich der anhaltende Erfolg in Lateinamerika (zur Aktualität dort vgl. Agudelo B. [Anm.4], S.Xff.; Llobet R. [Anm.8], S. 84 ff.; Beiderman [Anm.4], S.414ff.; Da Costa [Anm. 4], S. 420 f.), während europäische Autoren geteilter Meinung über die heutige Bedeutung Beccarias sind; vgl. etwa Küper, JuS 1968, 553 re. Sp., für den die „Aktualität der Gedanken“ sich auch heute noch „zu einer lohnenden Lektüre" macht und de Faria Costa (Anm. 4), 104, für den es „zwingend“ (imperioso) ist, Beccaria heute zu lesen, während für Vormbaum (Anm. 8), S. 317 Beccaria „dem heutigen Strafrecht nicht mehr viel zu sagen hat“. 
hat und zwar in ideeller wie materieller Hinsicht ${ }^{59}$. So erklärt sich auch, dass er selbst es - wie ein eigenes Werk - verteidigt hat ${ }^{60}$.

Was das Thema der Folter angeht, so ist zunächst um der historischen und wissenschaftlichen Redlichkeit willen darauf hinzuweisen, dass Beccaria keineswegs der erste war, der sich gegen die Folter gewendet hat ${ }^{61}$. Jerouschek hat Beccaria insoweit vorgeworfen, dass er Friedrich von Spees Cautio criminalis (1631) nicht erwähnt habe, obwohl er sie gekannt haben müsse und sich darin schon „fast alles“ finde, was Beccaria zur „Widerlegung“ der Folter zu sagen hatte $^{62}$. Auch ein Hinweis auf Thomasius' Dissertatio de tortura ex foris christianorum proscribenda (1705) findet sich bei Beccaria nicht ${ }^{63}$. Vor allem aber hat er nie die schon erwähnte intellektuelle (Mit-)Urheberschaft Pietro Verris an den Deliti gerade hinsichtlich der Folter anerkannt, obwohl dieser praktisch zeitgleich ${ }^{64}$ an seinen „Osservazioni sulla tortura“ gearbeitet, diese drei Jahre später (1777) beendet hat und sie im Jahre 1804 veröffentlicht wurden ${ }^{65}$. So ist es wenig verwunderlich, dass sich in den Osservazioni zahlreiche implizite Hinweise auf Beccaria finden, die ein etwas trübes Licht auf seine intellektuelle Redlichkeit werfen, weil in ihnen der Vorwurf mitschwingt, dass Beccarias Argumente gegen die Folter in Wirklichkeit alter Wein in neuen Schläuchen war ${ }^{66}$. Mögen es auch

59 Vgl. Malarino (Anm. 25), S. 215 f., wo er in Fn. 148 insbesondere den Brief Pietro Verris an die Mailänder Freunde zitiert: „als er das Material beisammen hatte, schrieb ich es nieder und brachte es in eine Ordnung und machte ein Buch daraus“; sowie Fn. 150, wo er den Brief Alessandro Verris an Isidoro Bianchi zitiert, aus dem sich ergibt, dass Beccaria die „erforderliche Ausdauer“ zur Abfassung des Werks fehlte und täglich nicht mehr als zwei Stunden daran arbeiten konnte, wobei Pietro Verri „sich die Mühe machte, das Werk mit eigener Hand in eine Endfassung zu bringen." Vgl. auch ebd., S. 218 mit Fn. 154 und S. 219 mit Fn. 156 mit Nachweisen zur späteren Korrespondenz der Gebrüder Verri, wo als Reaktion auf die fehlende Erkenntlichkeit Beccarias sogar das Wort „Plagiator“ fällt (Fn. 154) und Pietro einen „Krieg“ gegen das Buch vor allem deshalb ablehnt, weil es „auf unsere Anregung hin verfasst“ wurde und „zu dessen Entstehen wir so viel geholfen haben." Vgl. auch schon Llobet $R$. (Anm. 8), S. 68 („... en general los biógrafos de Beccaria consideran que fue escrita por los hermanos Verri, principalmente por Pietro“); Barbero Santos (Anm. 3), 140 f., 147 (insbesondere 141: wonach Pietro den Text „nicht nur in Reinschrift übertragen, sondern ihn auch überarbeitet hat" [„no sólo lo pasó a limpio, sino que ... lo reelaboró ..."]); Pisapia (Anm. 52), S. 55. Dagegen betont Zaffaroni (Anm.4), S. 423, dass das Verdienst des Buchs und seine Kreativität Beccaria zustehe; die insoweit geäußerte Kritik hält er für ein Produkt von antiaufklärerischen Beccaria Gegnern (insoweit auch S. 443 f.).

60 Malarino (Anm. 25), S. 216 f. mit Fn. 151 f.; Llobet R. (Anm. 8), S. 68.

61 Vgl. auch Malarino (Anm. 25), S. $195 \mathrm{ff}$.

62 Jerouschek, ZStW 110 (1998), S. 672 f. Zu von Spee auch Schulz, Die Ausweitung des Folterbegriffs unter menschlichen Aspekten, in: Jacobs (Anm. 8), S. 225 ff., der schon bei von Spee Nützlichkeitsargumente ausmacht (ebd., S. 229).

63 Vgl. Jerouschek, ZStW 110 (1998), S. 671 f., der Thomasius aber nicht als Wegbereiter Beccarias sieht; zu Thomasius auch Schulz (Anm.62), S. 230 ff. Für einen Vergleich mit der ein Jahr später erschienen Schrift Sonnenfels vgl. Cattaneo (Anm. 8), S. $49 \mathrm{ff} ., 54 \mathrm{ff}$.

64 Nach Malarino (Anm. 25), S. 179 f., gehen viele der Reflexionen und Gedanken der Osservazioni „auf eine frühere Zeit zurück, insbesondere auf diejenige der ,Accademia die Pugni' und der Zeitschrift Il Caffe.“

65 Zur Entstehungsgeschichte genau Malarino (Anm. 25), S. 175 ff. Pietro Verri starb im Jahr 1797, so dass sein Werk von seinem Assistenten Pietro Custodi veröffentlicht werden musste. Vgl. Malarino (Anm. 25), S.197, 219 (unter Verweis auf eine unveröffentlichte Rezension 
die Autoren der Aufklärung mit wissenschaftlicher Redlichkeit nicht so genau genommen haben ${ }^{67}$, wie wir das heute zu tun verpflichtet $\operatorname{sind}^{68}$, und mag auch „der kriminalwissenschaftliche Diskurs der Aufklärung kaum durch die Kreation neuer Argumente imponiert" haben, sondern seine epochemachende Leistung in der „Säkularisierung der Moral“ gelegen haben ${ }^{69}$, und darf man schließlich sicher nicht das persönliche Risiko, das Beccaria mit der Veröffentlichung unter seinem Namen auf sich genommen hat, geringschätzen, so hinterlässt doch jedenfalls die vollständige Unterschlagung jeglicher aufklärerischer Tradition $^{70}$ hinsichtlich der Ablehnung der Folter und insbesondere des Beitrags des ihm besonders nahestehenden Verri einen bitteren Nachgeschmack.

\section{Beccarias Argumente gegen die Folter}

Wenn wir uns nun den eigentlichen Argumenten Beccarias gegen die Folter zuwenden, so lassen sich dem einschlägigen Textteil ${ }^{71}$ neun Argumente gegen die Folter mit unterschiedlicher Überzeugungskraft entnehmen:

1. Die Folter stelle eine vorweggenommene Strafe und zugleich Verletzung der Unschuldsvermutung dar, weil ohne vorherige Feststellung der Schuld gerade zu deren Feststellung gefoltert würde und dies eben auch Unschuldige treffe ${ }^{72}$. Die Gefahr, dass Unschuldige gequält würden, sei hoch, weil die Mehrzahl der Bürger - ganz im Sinne negativer Generalprävention - die Gesetze befolgten, also unschuldig $\operatorname{sei}^{73}$.

2. Die Folter verletze das Selbstbelastungsverbot, weil der „Ankläger zugleich Angeklagter“ sei, sich der Gefolterte eben selbst belasten müsse ${ }^{74}$.

3. Die Folter sei „schändlicher Prüfstein der Wahrheit“ („infame crociuolo della verità “) und irrational, weil sie den widerstandsfähigen, schmerzunempfindlichen Starken gegenüber dem Schwachen privilegiere und damit ebenso

Pietro Verris, in welcher dieser die geringe Originalität des Werks kritisierte), S. 220 („im eigenen Text viele jener Argumente benutzt hatte, ohne die Autoren anzugeben.").

67 Jerouschek, ZStW 110 (1998), S. 672.

68 Vgl. die Empfehlungen der DFG Kommission zur "Selbstkontrolle in der Wissenschaft“ von 1998 unter http://www.dfg.de/download/pdf/dfg_im_profil/reden_stellungnahmen/ download/empfehlung_wiss_praxis_0198.pdf. Schon unter Empfehlung 1, S. 7 lesen wir dort, dass es zu den allgemeinen Prinzipien wissenschaftlichen Arbeitens gehört, „Resultate zu dokumentieren“ und „strikte Ehrlichkeit im Hinblick auf die Beiträge von Partnern, Konkurrenten und Vorgängern zu wahren“.

69 Jerouschek, ZStW 110 (1998), S. 672.

70 Immerhin zitiert er Montesquieu (Beccaria, Von den Verbrechen [Anm. 1], S. 28), allerdings in Zusammenhang mit geheimen Anklagen (Kap. IX).

71 Kap. XVI der hier verwendeten Übersetzung von Vormbaum (Anm. 1).

72 Beccaria, Von den Verbrechen (Anm.1), S. 31.

73 Ebd., S. $31 \mathrm{f}$.

74 Ebd., S. 32. 
irrational sei wie der mittelalterliche Gottesbeweis ${ }^{75}$ : „Von zwei gleichermaßen unschuldigen oder gleichermaßen schuldigen Menschen wird der starke und mutige freigesprochen ..., der schwache und ängstliche verurteilt ..." ${ }^{\text {76 }}$.

4. Die Folter privilegiere den Schuldigen gegenüber dem Unschuldigen, denn während dieser „alle Möglichkeiten gegen sich“ habe - entweder gestehe er und werde deshalb bestraft oder er werde für unschuldig erklärt, habe aber die „nicht geschuldete Strafe“ der Folter erlitten -, habe jener immerhin die Chance, wenn er der Folter widerstehe, ungerechtfertigterweise freigesprochen zu werden und damit „eine größere Strafe gegen eine geringere“ einzutauschen ${ }^{77}$.

5. Die Folter vernichte - wie der Gottesbeweis - die Willensfreibeit, denn der Schmerz lasse dem Gefolterten keine andere Wahl, als „sich als Schuldiger zu bezeichnen“, um „damit der Folter zu entkommen“78.

6. Die Folter produziere damit zugleich Unwabrbeit, denn das Geständnis ist nicht Ausdruck der Wahrheitsliebe des Gefolterten, sondern Folge der Pein und seines Wunsches dieser zu entkommen ${ }^{79}$. Viele Unschuldige haben sich „wegen der Qualen der Folter für schuldig bekannt ..." ${ }^{80}$. Außerdem sei der Wahrheitsgehalt einer Aussage beim Gefolterten noch weniger erkennbar als bei einem ruhigen Menschen.

7. Die Anwendung von Folter zur Reinigung von Ehrlosigkeit - auf religiösen und geistigen Bräuchen beruhend - sei „lächerlich“, weil damit neue Ehrlosigkeit hervorgebracht würde, nämlich die des Folteropfers ${ }^{81}$.

8. Die Folter erweise sich als Würdeverletzung, wie sie nur gegenüber NichtPersonen, z. B. den Sklaven im alten Rom, vorkomme ${ }^{82}$.

9. Sei die Folter schon ungeeignet zur Feststellung der Tat des Gefolterten, so könnten noch weniger andere Taten und/oder Beteiligte entdeckt werden. Mehr noch: Der Gefolterte werde andere noch leichter als sich selbst anklagen ${ }^{83}$. Es sei ungerecht, Menschen wegen Verbrechen anderer zu foltern. Es sei auch unnütz, weil diese auf diese Weise nicht entdeckt würden und meist schon geflohen $\operatorname{seien}^{84}$.

Ebd., S. 32 f., 33 f.; im Original (Anm. 1), S. 22.

Beccaria, Von den Verbrechen (Anm. 1), S. 33.

Ebd., S. 34 f. Vgl. auch S. 29 (Kap. X. Suggestivbefragungen. Aussagen): „.. der Schmerz wird dem Starken ein hartnäckiges Schweigen suggerieren, weil er so die größere Strafe gegen die kleinere eintauscht, dem Schwachen aber wird sie das Geständnis suggerieren, weil er sich damit von dem gegenwärtigen Schmerz befreit, der für den Augenblick wirksamer ist als der zukünftige Schmerz."

Ebd., S. 33 .

Ebd., S. 33 .

Ebd., S. 35 .

Ebd., S. 36 ff.; allgemein zur Ehrlosigkeit ebd., S. 59.

Ebd., S. 38.

Ebd., S. 36.

Ebd., S. 36. 
Zusammenfassend: Die Folter provoziere unwahre Aussagen und privilegiere die Starken und Schuldigen gegenüber den Schwachen und Unschuldigen (Argumente 3, 4,6), sie führe zur Verfolgung Unschuldiger wegen eventuell erfundener Taten (Argument 9). Beccaria erkennt zwar zugleich die Ungerechtigkeit der Folter mit Blick auf Unschuldsvermutung ${ }^{85}$, Selbstbelastungsverbot und Willensfreiheit an (Argumente 1,2,5), er sieht sogar ihren entwürdigen und entehrenden Charakter (Argumente 7, 8), doch ergibt sich - abgesehen von offensichtlichen Widersprüchen ${ }^{86}$ - aus dem Zusammenhang seiner Argumentation mit seiner oben dargestellten utilitaristischen und zweckorientierten Strafrechtskonzeption, dass er die Folter nicht an sich für böse hält - die von ihm vorgeschlagenen Freiheitsstrafen sind in der Tat mindestens genau so grausam ${ }^{87}$ -, sondern nur für unnütz und damit schädlich für ein effizientes Strafrecht ${ }^{88}$. Alles in allem lehnt Beccaria die Folter damit vorrangig aus utilitaristischen und nur sekundär aus humanitären Gründen $a b^{89}$.

Bemerkenswert ist überdies, dass Beccaria den Zusammenhang zwischen Folter und mittelalterlichem Strengbeweis, insbesondere Geständniszwang ${ }^{90}$, nicht gesehen zu haben scheint ${ }^{91}$, obwohl er an anderer Stelle ${ }^{92}$ in bewusster Distanzierung von der mittelalterlichen Beweismittelarithmetik zwischen „vollständigen und unvollständigen Beweisen“ unterscheidet und eine „moralische Gewißheit“" (morale certezza“) ${ }^{93}$ auf der Grundlage eines Wahrscheinlichkeitsurteils für eine Verurteilung für ausreichend hält.

Es bleibt also dabei: Beccarias Humanismus, wenn man ihn den in seinem Werk zu entdecken glaubt, wird den Notwendigkeiten eines gesellschaftsschützenden Strafrechts untergeordnet.

Cattaneo (Anm. 8), S. 60 sieht in der „Verteidigung der durch die Einrichtung der Folter bedrohten Unschuld“ das zentrale Argument der „juristischen Aufklärung“. H. SchülerSpringorum, KritV 1991, 123 (in englisch in: Centro Nazionale [Anm. 4], S. 121 ff.), 132 sieht im Zusammenhang mit dem inquisitorischen Strafverfahren der Zeit Beccarias den „realen Fortschritt“ des Werks in „einer durchgängigen Betonung des Rechts des Beschuldigten, sich selbst zu entlasten ..."

86 So lehnt er z. B. das Selbstbelastungsverbot an anderer Stelle ab; vgl. Anm. 32.

87 Vgl. schon Anm. 32.

88 Ebenso Naucke, in: Deimling (Anm. 7), S. 48. Voltaire, Commentaire sur le livre des délits et des peines, par un avocat de province, 9. Aufl. 1767, abrufbar unter http://books.google.de, S. 63 scheint das anders zu sehen, wenn er Beccaria bezüglich seiner Ausführungen zur Folter als „Liebhaber der Menschheit“ („amateur de l'humanité") bezeichnet.

89 Ebenso Naucke, in: Deimling (Anm. 7), S. 48. Anders z. B. Voltaire (Anm. 88), S. 60, für den die Ablehnung der Folter vorrangig eine Herzensangelegenheit ist: „... tous cependant, para une pitié que Dieu a mise dans nos cœurs, s'élévent contre les tortures qu'on fait souffrir aux accusés don on veut arracher l'aveu."

90 Dazu Ambos, Jura 2008, $589 \mathrm{f}$.

91 Krit. insoweit auch Jerouschek, ZStW 110 (1998), S. 670 f.

92 Beccaria, Von den Verbrechen (Anm. 1), Kap. VII. Indizien und Verfahrensformen, S. 20 ff. (im Original [Anm. 1], Kap. XIV. Indizi, e forme di giudizi).

93 Dei delitti (Anm. 1), S. 19. Die Übersetzung von „certezza“ mit „Gewißheit“ (so z. B. auch die Übersetzungen von Esselborn [Anm. 1], S. 79 und Hommel [Anm. 3], S. 73) ist „Beweissicherheit" (Vormbaum [Anm. 1], S. 21) vorzuziehen. 


\section{Zur heutigen Bedeutung von Beccarias Argumentation}

Wenn man nun Beccarias Argumente gegen die Folter aus heutiger Sicht Revue passieren lässt, so sind sie einerseits in ihrer primär utilitaristischen Ausrichtung überholt und andererseits zu sehr auf die grausame (repressive) Überführungsfolter des inquisitorischen Strafverfahrens fixiert, um Antworten auf die diffizileren Herausforderungen des Folterverbots in unserer Zeit liefern zu können. Heute wird das Folterverbot nicht länger zweckgebunden verstanden, sondern es ist Ausfluss des unverbrüchlichen Schutzes der Menschenwürde ${ }^{94}$ und als solches in internationalen und regionalen Menschenrechtsverträgen sowie im humanitären Völkerrecht verankert ${ }^{95}$. Das Folterverbot gilt absolut (also auch im Ausnahmezustand) ${ }^{96}$ und ist zwingendes Völkerrecht (ius cogens) ${ }^{97}$. Seine Verletzung kann als Verbrechen gegen die Menschlichkeit oder Kriegsverbrechen geahndet werden ${ }^{98}$. Die Effizienzargumentation gegen die Folter ist damit zwar nicht abgeschafft - insoweit ist Beccarias Argumentation nicht vollkommen überholt -, sie tritt aber hinter der Menschenwürdeargumentation zurück.

Beccaria musste sich allerdings nicht mit den daraus erwachsenden Definitionsproblemen herumschlagen, nicht nur wegen der damals noch fehlenden

94 Vgl. Supreme Court of Israel, Public Committee Against Torture et al. vs. State of Israel et al., Urteil vom 6. September 1999 (abgedruckt in: S. Levinson [Hrsg.], Torture: A Collection, 2004, S. 165 ff.), para. 22; M. Delmas-Marty, Journal of International Criminal Justice (JICJ) 5 (2007) S. 584, 592 (Folterverbot als Ausdruck des universellen Werts der Menschenwürde); Hassemer, Süddeutsche Zeitung vom 27.2. 2005, auch abgedruckt in Erscheinungsformen des modernen Rechts, 2007, S. 17 („Folter ist, wenn ich dem Menschen mit Gewalt seine Autonomie nehme, ihn zu einem bloßen Körper mache.“); A. Eser, Festschrift für Hassemer, 2010, S. 715 („zum entpersönlichten Objekt äußerer Fremdregie erniedrigt“).

95 Siehe vor allem Art. 7 Int. Pakt für bürgerliche und politische Rechte, Art. 2 UN-Folterkonvention, Art. 3 EMRK, Art. 5(2) AMRK; bezüglich des humanitären Völkerrechts siehe den gemeinsamen Art. 3 (1) der Vier Genfer Konventionen (GK) von 1949 sowie Art. 17(4) Dritte Genfer Konvention (GK III), Art. 32 GK IV, Art. 75(2)(ii) Zusatzprotokoll I und Art. 4(2)(a) Zusatzprotokoll II GK. Ausführlich zum Folterverbot im europäischen und Völkerrecht M. Möblenbeck, Das absolute Folterverbot etc., 2008, S. 39 ff., S. 56; siehe auch für die internationalen Folterverbote J.P. Polzin, Strafrechtliche Rechtfertigung der ,Rettungsfolter'? etc., 2008, S. 59 ff.; J. Kümmel, Eine rechtliche Betrachtung der Folter als Mittel der Gefahrenabwehr unter besonderer Berücksichtigung des Nötigungstatbestandes, 2008, S. 50 ff.; A.K. Weilert, Grundlagen und Grenzen des Folterverbotes in verschiedenen Rechtskreisen, 2009, S. 55 ff., 65 f.

96 ' $\mathrm{N}] \mathrm{o}$ exceptional circumstances whatsoever, whether a state of war or a threat or war, internal political instability or any other public emergency, may be invoked as a justification of torture.' (Article 2 UN-Folterkonvention). Dies wird bestätigt durch Entscheidungen des Committee against Torture, z.B. die Entscheidung über Belgien vom 27. Mai 2003, CAT/ $\mathrm{C} / \mathrm{CR} / 30 / 6$. In dieser Entscheidung empfahl das Committee Belgien eine Klausel im Strafgesetzbuch einzuführen, die den Notstand als Rechtfertigungsgrund in Folterfällen ausdrücklich ausschließt.

97 Vgl. grundlegend International Criminal Tribunal for the Former Yugoslavia (ICTY), Prosecutor v. Furundija (IT-95-17/1-T), 10 December 1998, \$\$153-157. Siehe auch E. Benvenisti, European Journal of International Law (EJIL) 8 (1997), S. 596, 603 mit Fn. 42); P. Gaeta, JICJ 2 (2004) S. 785, 787.

98 Vgl. Art.7 (1) (f) und Art. 8(2) (a) (ii), (c)(ii) IStGH-Statut. 
normativen Absicherung des Folterverbots, sondern vor allem weil die von ihm ins Visier genommen Folterpraxis an Grausamkeit kaum zu überbieten war und insoweit ohne weiteres einem an der Menschenwürde orientierten Folterbegriff - im Sinne der qualifizierten Objektformel des Bundesverfassungsgerichts ${ }^{99}$ subsumiert werden konnte. Heute aber entstehen mitunter schwierige, schon in den einschlägigen völkerrechtlichen Definitionen ${ }^{100}$ angelegte Abgrenzungsprobleme etwa mit Blick auf nötigende Vernehmungsmethoden (vgl. nur \136a StPO) ${ }^{101}$, die eben nicht ohne weiteres als Folter qualifiziert werden können. Der Europäische Gerichtshof für Menschenrechte differenziert nach den verschiedenen degrees of injury, also dem Verletzungsgrad, zwischen torture, mistreatment und ordinary treatment ${ }^{102}$. In der grundlegenden Entscheidung des israelischen Supreme Court von $1989 \mathrm{zu}$ „robusten“ Vernehmungsmethoden im Rahmen von ticking-bomb Fällen ${ }^{103}$ wurden ziemlich alle vorstellbaren Vernehmungstechniken detailliert untersucht und bewertet ${ }^{104}$. So befasst sich das Gericht beispielsweise mit dem handcuffing. Der Tatverdächtige wird für die Dauer der Vernehmung mit Handschellen an Armen und Beinen gefesselt. Bei einer anderen Befragungstechnik wird dem Verdächtigen ein Sack über den

99 BVerfGE 30, 1, 25 f.: „Allgemeine Formeln wie die, der Mensch dürfe nicht zum bloßen Objekt der Staatsgewalt herabgewürdigt werden, können lediglich die Richtung andeuten, in der Fälle der Verletzung der Menschenwürde gefunden werden können.... Eine Verletzung der Menschenwürde kann darin allein nicht gefunden werden. Hinzukommen muss, dass er einer Behandlung ausgesetzt wird, die seine Subjektqualität prinzipiell in Frage stellt, oder das in der Behandlung im konkreten Fall eine willkürliche Missachtung der Würde des Menschen liegt." Im Fall Daschner hat das BVerfG (NJW 2005, 656) mit Blick auf (die Androhung von) Folter festgestellt, dass damit „die Vernehmungsperson zum bloßen Objekt der Verbrechensbekämpfung unter Verletzung ihres verfassungsrechtlich geschützten sozialen Wert- und Achtungsanspruch" gemacht und ,grundlegende Voraussetzungen der individuellen und sozialen Existenz des Menschen " zerstört werden.

100 Siehe Anm. 95 und Ambos, JICJ 6 (2008), S. 261, 265 f.

101 Die in $\$ 136 a$ Abs. 1 aufgeführten Vernehmungsmethoden (Misshandlung, Ermüdung, Quälerei etc., sog. „Verhör dritten Grades“) sind nicht abschließend; Diemer, in: Karlsruher Kommentar zur StPO, 6. Aufl. 2008, \$136a Rdn. 9; entscheidend ist, ob diese oder andere „Vernehmungstechniken“ die Willensfreiheit beeinträchtigen. Zur Folter in diesem Zusammenhang siehe BVerfG (Anm. 99).

102 Siehe EGMR, Ireland v. UK, Urteil vom 18. 1.1978 (application n ${ }^{\circ}$ 5310/71), \$167: „Although the five techniques, as applied in combination, undoubtedly amounted to inhuman and degrading treatment, although their object was the extraction of confessions, the naming of others and/or information and although they were used systematically, they did not occasion suffering of the particular intensity and cruelty implied by the word torture as so understood." Siehe auch Benvenisti, EJIL 8 (1997), S. 604 f.; Y. Shany, Catholic University Law Review 56 (2007) S. 101, $118 \mathrm{f}$.

103 In diesen Fällen haben die Ermittler einen Tatverdächtigen festgenommen, von dem sie glauben, er gehöre zu einer terroristischen Organisation und habe Informationen über bestimmte Anschläge, wisse beispielsweise, dass eine bestimmte Bombe zu einem bestimmten Zeitpunkt explodiere. Nur unter Anwendung von Zwangsmaßnahmen können sie die Informationen erhalten und so das Leben Unschuldiger retten. Das ist ein klassisches Beispiel heutiger Terrorismusbekämpfung und spiegelt häufig die Realität in Israel - und nicht nur dort! wieder. Für Beispiele siehe Y. Ginbar, Why Not Torture Terrorists? Moral, practical, and legal aspects of the ,ticking bomb' justification for torture, 2008, S. $357 \mathrm{ff}$.

104 Supreme Court of Israel (Anm. 94). 
Kopf gestülpt, so dass er den Vernehmenden nicht sehen kann. Alle diese Maßnahmen, schwerer oder leichter Art, werden untersucht und differenziert betrachtet. Der Supreme Court gelangt z. B. zu dem Ergebnis, dass das handcuffing als isolierte Maßnahme keine Folter ist ${ }^{105}$. Vielmehr würden die Rechte des Vernehmungsbeamten geschützt, indem Übergriffen durch den Tatverdächtigen vorgebeugt würden. Allerdings könne die kombinierte Anwendung mehrerer an sich zulässiger Maßnahmen, ihr combined effect, Folter darstellen ${ }^{106}$. In der Literatur wird die Relevanz dieser Definitionsbemühungen teilweise bestritten. $\mathrm{Zu}$ überzeugen vermag dies jedoch nicht. Denn wenn Folter absolut verboten sein soll, einfache Misshandlung aber keine Folter ist und damit vom Verbot nicht erfasst ist, dann muss Folter von der einfachen Misshandlung abgegrenzt werden ${ }^{107}$. Diese Abgrenzung kann nicht abstrakt, sondern nur fallbezogen erfolgen ${ }^{108}$.

Wenn man nun bei bestimmten „robusten“ Vernehmungsmethoden davon ausgeht, dass die Folterschwelle überschritten ist, so stellt sich die weitere Frage, ob diese Methoden auch in Extremsituationen, wie etwa den gerade genannten ticking-bomb Fällen ${ }^{109}$ oder dem Entführungsfall Gäfgen/von Metzler ${ }^{110}$, verboten sein sollen, in Situationen also, in denen Folter - als sog. (präventive) Rettungsfolter - zur Erlangung lebensrettender Informationen eingesetzt wird.

105 Ebd., para. 26.

106 Ireland v. UK (Anm. 102); Supreme Court of Israel (Anm. 94), para. 30: „Their combination, in and of itself gives rise to particular pain and suffering."

107 Zutreffend auch Eser, Festschrift für Hassemer, 2010, S. 716 f., 719.

108 In Bezug auf das erforderliche „minimum level of severity“ sagt der EGMR Folgendes: „[it] is, in the nature of things, relative; it depends on all the circumstances of the case, such as the duration of the treatment, its physical or mental effects and, in some cases, the sex, age and state of health of the victim, etc"; Ireland v. UK (Anm. 102), para. 162. Zur Ansicht des UN-Human Rights Committee (Menschenrechtsausschuss) siehe Benvenisti, EJIL 8 (1997), S. 606. Nach R. Merkel, Festschrift für Günther Jakobs, 2007, S. 375, 398 ff., hängt das Folterkonzept (auch) von den betroffenen Rechten ab.

109 Siehe Anm. 103.

110 Der Jurastudent Markus Gäfgen entführte Jakob von Metzler, den 11-jährigen Sohn eines Bankiers aus Frankfurt, um Lösegeld zu erpressen. Bei der Geldübergabe wurde er festgenommen und anschließend verhört, weigerte sich jedoch, den Aufenthaltsort des Jungen preiszugeben. Nach einem Tag erfolgloser Befragung befahl Polizeivizepräsident Daschner einem Untergebenen, Gäfgen mit der Zufügung von Schmerzen zu drohen, wenn er nicht endlich sagen würde, wo er den Jungen versteckt hielt (LG Frankfurt, Urteil vom 20. Dezember 2004, abgedruckt in NJW 2005, S. 692-696, 692 re. Sp.). Daschner sah hierin die einzige Möglichkeit, das Leben von Jakob zu retten. Die Drohung zeigte Wirkung. Gäfgen kooperierte, die Polizei konnte allerdings nur noch die Leiche des Jungen bergen. Der Fall hatte mehrere Strafverfahren zu Folge. Daschner und sein Untergegebener wurden unter anderem wegen Nötigung, Gäfgen wegen Mordes verurteilt (LG Frankfurt, Urteil vom 9. April 2003, abgedruckt in StV 2003, 325; bestätigt in der Revision, siehe BGH, Urteil vom 21. Mai 2004, 2 StR 35/04 und BVerfG, Urteil vom 14. Dezember 2004, 2 BvR 1249/04 = NJW 2005, 656). Der EGMR hat im Rahmen der üblichen Gesamtbetrachtung (vgl. Ambos, Internationales Strafrecht, 2. Aufl. 2008, $\$ 10$ Rdn. 34 f.) - das Verfahren nicht als insgesamt unfair beurteilt, insbesondere weil das erstinstanzliche LG Frankfurt (natürlich) die Verwertung des abgepressten Geständnisses und darauf beruhender Aussagen für unverwertbar $(\$ 136 a$ StPO) hielt (Gäfgen v. Deutschland, Urteil vom 30.6.2008 - No. 22978/05 = NStZ 2008, 699, para. 97 ff. m.w.N.). 
Die Frage ist, wie schon anderen Orts dargelegt ${ }^{111}$, zu Recht sehr kontrovers und kann nicht mit einem Generalverweis auf das absolute Folterverbot erledigt werden $^{112}$. Denn man kann schon mit Fug und Recht bezweifeln, ob ein Handeln mit Rettungswillen überhaupt als Folter qualifiziert werden kann, jedenfalls wenn man dafür eine verabscheuungswürdige $Z$ weckrichtung verlangt ${ }^{113}$. Vor allem aber kann schwerlich von entpersönlichender Willkür i.S.d. qualifizierten Objektformel ${ }^{114}$ gegenüber dem Tatverdächtigen die Rede sein, wenn Folter zur Rettung eines oder mehrerer Menschen aus konkreter Lebensgefahr eingesetzt wird, es also gerade um die Verteidigung der Menschenwürde der Opfer des Folteropfers geht, und dieses doch dadurch gerade in seiner mitmenschlichen Verantwortlichkeit ${ }^{115}$ ernst genommen wird ${ }^{116}$. Selbst wenn man so weit nicht zu gehen bereit ist, weil man jedenfalls in der Folter immer eine Behandlung des Folteropfers als Sache, zumal zum Zwecke der Rettung eines Dritten, also instrumentalisiert, sieht ${ }^{117}$, dieses damit also doch „zum bloßen Objekt der Staatsgewalt herabgewürdigt" wird ${ }^{118}$, so darf doch nicht außer Acht gelassen werden, dass auch die Würde des (anderen) Opfers auf dem Spiel steht und der Staat sich (auch) insoweit nicht gleichgültig verhalten kann ${ }^{119}$. Deshalb ist alles in allem eine differenzierte Betrachtung gefordert, die an der Absolutheit des Folterverbots mit Blick auf die völkerrechtliche Staatenverantwortlichkeit festhält, aber hinsichtlich der individuellen Verantwortlichkeit des Folterers zwischen der Rechtswidrigkeit seines Verhaltens und seiner Schuld unterscheidet ${ }^{120}$. Die darin zum Ausdruck kommende Dichotomie zwischen Staat und Individuum spiegelt sich in der Dichotomie von einerseits staatsorientierten Menschenrechten/humanitärem Völkerrecht und andererseits individualrechtlichem Völkerstrafrecht wieder. Während jene die Absolutheit des Folterverbots verteidigen, zeigt sich dieses flexibler, indem es Gründe zum Ausschluss strafrechtlicher Verantwortlichkeit anerkennt ${ }^{121}$. In den uns beschäftigenden Extremfällen kommt zwar eine

111 Vgl. Ambos, JICJ 6 (2008), S. 261 ff. m.w.N.; zusf. ders., Festschrift für Fritz Loos, 2010.

112 So aber Delmas-Marty, JICJ 5 (2007), S. 592, die einen Strafausschluss in Fällen der Rettungsfolter mit einem allgemeinen Verweis auf die Menschenrechte als Auslegungsmaßstab i. S.v. Art. 21 (3) IStGH-Statut ablehnen will.

113 Vgl. etwa Eser, Festschrift für Hassemer, 2010, S. 719.

114 Siehe Anm. 99.

115 Sofern man von „Verantwortlichkeit“ mit Blick auf Leben, körperliche Unversehrtheit und/ oder Freiheit seines Opfers - entgegen (der Fiktion) der Unschuldsvermutung - in concreto wird ausgehen können. Also: Natürlich ist das Folteropfer grundsätzlich nur Tatverdächtiger und kann sich als solcher auf die Unschuldsvermutung berufen, aber das schließt doch nicht per se seine Verantwortlichkeit für Wohl und Wehe seines Opfers aus. Letztlich hängt das vom konkreten Fall ab.

116 Überzeugend Eser, Festschrift für Hassemer, 2010, S. 719 f.

117 Also im Sinne Kants (Anm.11).

118 Eben im Sinne der qualifizierten Objektformel (Anm. 99).

$119 \mathrm{Zu}$ dieser „Würdekollision“ näher Ambos, JICJ 6 (2008), S. 277 m.w.N. in Fn. 99 f. Krit. aber jüngst F. Lamprecht, Darf der Staat foltern, um Leben zu retten?, 2009, S. $113 \mathrm{ff}$

120 Näher Ambos, JICJ 6 (2008), S. 272 ff.

$121 \mathrm{Zu}$ dieser Dichotomie siehe auch Gaeta, JICJ 2 (2004), S. 789 f.; siehe ähnlich Shany, Catholic University Law Review 56 (2007), S. 126 ff., der das strikte humanitäre Völkerrecht der „Re- 
Rechtfertigung wegen ihrer objektiven, unrechtsaufhebenden Wirkung nicht in Betracht $^{122}$, dem Vernehmungsbeamten kann die Anwendung der Folter aber jedenfalls dann nicht vorgeworfen werden, wenn er sich in einer moralischen Zwangslage befunden und sie erst nach gründlicher Prüfung als letztes Mittel zur Rettung unschuldiger Menschen eingesetzt hat. Dann sollte er - wie immer man dies auch dogmatisch begründet - entschuldigt werden. Im Hinblick auf die individuelle strafrechtliche Verantwortlichkeit bei Rettungsfolter - aber nur insoweit! - kann das Folterverbot also nicht absolut gelten ${ }^{123}$.

lativität" des Völkerstrafrechts gegenüberstellt. Benvenisti, EJIL 8 (1997), S.609, sieht 'incoherence between the international and national spheres'.

122 Für eine Rechtfertigung aber grundsätzlich Eser, Festschrift für Hassemer, 2010, S. 723 ff., weil „einem Retter in Not nicht zugemutet werden“ könne, „etwas Unrechtes zu tun.“

123 Zur absoluten prozessualen Geltung mit der Folge eines Verwertungsverbots bzw. einer exclusionary rule siehe Ambos, $\mathrm{StV}$ 2009, $151 \mathrm{ff}$.; grds. ders., Beweisverwertungsverbote, 2010, S. $99 \mathrm{ff}$. 